\section{P2-358 HIV/AIDS STATUS IN A HILL-TRIBE POPULATION, THAILAND}

doi:10.1136/jech.2011.142976k.90

T Apidechkul.. Mae Fah Luang University, Chiang Rai Province, Thailand

Introduction We aimed to describe the current HIV/AIDS situation, sexual behaviours, access to care and survival time in the six main hill-tribe people in Thailand; Akha, Lahu, Karean, Mong, Yao, and Lisaw This is where much of the epidemic of HIV/AIDS in Thailand occurs.

Materials and Methods Using a retrospective cohort study design we systematically extracted data from the medical records of hill-tribe HIV/AIDS patients from a total of 37 hospitals. We also studied risk factors by administering a questionnaire delivered by fact-to-fact interview. The questionnaires had been tested for validity and reliability. Statistic analyses were contribute by Survival Analysis and Cox-Regression

Results A total of 608 cases came from 37 hospitals. Only 581 cases were included in the analysis. $64.9 \%$ of cases were female, $39.6 \%$ aged 26-35years at diagnosis, 94\% infected from their spouse, $22.6 \%$ were infected by sexual intercourse and $6.2 \%$ by IDU. $48.9 \%$ received ARV and $47.1 \%$ received OI drug. Survival analysis found that those who received ARV had a 50\% survival time of 12.4 years $(p<0.001)$ and those who received OI had a $50 \%$ survival time of 6.0 years $(p<0.001)$. Cox-Regression showed that having receiving ARV, OI, female sex, religion were factors favouring improved survival time. In addition, among men: 19\% gave a history of IDU, $63 \%$ had their first sexual experience below age 20 years, $23 \%$ had 2 or more partners, and $27 \%$ reported using condoms. Among females: 10\% had work as masseuses, and 3\% as sex workers

Conclusions This study shows that a given our knowledge of HIV/ AIDS there is scope for peer education among hill-tribe people, especially with regard to promoting condom use.

\section{P2-359 TOTAL AND CHRONIC DISEASE MEDIATED EFFECTS OF EDUCATIONAL ATTAINMENT ON MULTI-DIMENSIONAL HEALTH SCORE}

doi:10.1136/jech.2011.142976k.91

0 A Arah, ${ }^{*}$ C A Thompson. University of California, Los Angeles, California, USA

Introduction Education, chronic disease, and other factors such as occupation play a role in how one perceives and reports their health. The interrelationships of these factors are not well understood; specifically it is unclear how much of the education contribution can be explained through other factors such as chronic disease and whether this varies by world region and by gender. In this paper we quantified the total and mediated associations between educational attainment and multi-dimensional health score spanning eight health domains, across the world stratified by world region, national income, and gender.

Methods Using hierarchical regression methods to analyse World Health Survey data on 146561 individuals in 51 countries, we estimated the influence of education on a multidimensional health score that is calculated using self-reported assessments of eight global states of general health. We quantified both the total effects and effects not mediated by chronic disease status and occupation, with(out) stratification by world region, national income, and participant gender.

Results We found that individuals with no formal schooling consistently reported the lowest levels of health. How occupation and chronic disease mediated this association varied widely by world region and national income. Chronic disease status and occupation accounted for much more of this relationship in developed regions and higher income countries. On average, the results for women reflected an increased importance of education on health score compared with men.

Conclusion Western and wealthy countries and regions and women appear to be more successful at converting their educational attainment into better health.

\section{P2-360 THE INFLUENCE OF HEALTH STATE DOMAINS ON OVERALL SELF-REPORTED GENERAL HEALTH AMONG INDIVIDUALS IN 68 COUNTRIES}

doi:10.1136/jech.2011.142976k.92

0 A Arah, ${ }^{*}$ C A Thompson. University of California, Los Angeles, California, USA

Introduction Self-reported health is commonly used as an outcome in health and social science research, but how general health is related to specific health states or functional domains such mobility, affect and cognition remains unclear. Also, there is a paucity of research on whether, if any, such associations differ across world regions and countries. In this paper, we explore the influence of individual health domains, such as vision and mobility on selfreported general health, and how this influence may vary across world regions as categorised by geography, economy, and mortality. Methods Applying multilevel regression methods to World Health Survey data on 255000 individuals in 68 countries, we quantified associations between eight detailed health state domains (namely, mobility, self-care, pain, cognition, interpersonal activities, vision, sleep, and affect) and self-reported general health with(out) stratification by world region, national income grouping, and country mortality.

Results Overall, by region, and by income, levels of mobility and pain consistently had the most influence on general health score. For other health domains, similar patterns of influence were observed in Europe, the Americas, and the Middle East, but these patterns differed in Sub-Saharan Africa, Asia and the Pacific Islands. Differences in health state influence were also evident when comparing lower middle and low income countries with higher income countries.

Conclusion Although reduced mobility and increased levels of pain consistently predict self-assessed general health scores in all areas of the world, there are substantial differences, by geography and economic development, in how other health states impact general health.

\section{P2-361 DEMAND AND SUPPLY SIDE DETERMINANTS CAUSING LOW ORS AND ZINC USE RATE FOR DIARRHOEA TREATMENT AMONG CHILDREN IN UTTAR PRADESH (UP), INDIA}

doi:10.1136/jech.2011.142976k.93

G Arya. * UNICEF, Lucknow, Uttar Pradesh, India

Introduction Several studies have established the significance of ORS and Zinc in management of diarrhoea-ORS in terms of reducing the level of dehydration and Zinc for reducing the frequency, severity, duration and future episodes of diarrhoea - thereby contributing towards reducing child-mortality. The state of UP has an overall ORT use rate of only $29.2 \%$ in contrast to $88.4 \%$ in Meghalaya state and national average of $53.6 \%$.

Objective The main objective of this analysis is to identify the bottlenecks in prescription, accessibility, availability and utilisation of ORS and Zinc for management of diarrhoea in the community in the state of UP. 
Methods The analysis is based upon structured interviews conducted on a multi-district sample of 240 retailers/service providers and around 1500 households across UP. The data collected is then triangulated with other sources of data available for UP, collected within the reference period of 2008-2010. The location of retailers and service providers were then mapped to analyse the geographic spread and thus accessibility. This data are also supplemented with qualitative assessment of existing practices related to management of childhood diarrhoea.

Results and Conclusion Triangulation of data suggests that the following bottlenecks contribute to low ORS/Zinc use:

- Low awareness and perceived efficacy of ORS and Zinc for management of childhood diarrhoea both among service providers and end-users

- Erratic availability of ORS and Zinc in public-health facilities

- Geographic clustering of retailers/sources of ORS and Zinc

- Financial constraints of beneficiaries

'Coverage Evaluation Survey for India, UNICEF, 2009.

\section{P2-362 WITHDRAWN}

\section{P2-363 ASSOCIATION OF CLIMATE FACTORS WITH INCIDENCE DENGUE FEVER: AN ECO-EPIDEMIOLOGICAL ANALYSIS}

doi:10.1136/jech.2011.142976k.94

S Awan, ${ }^{*}$ M Tariq. Aga Khan University Hospital, Karachi, Pakistan

Background Dengue infection, which causes dengue fever, dengue haemorrhagic fever and dengue shock syndrome are currently endemic or intermittently epidemic in many tropical and subtropical regions of the world. According to the WHO, dengue has recently become a major public health concern globally. It is generally found in tropical and sub-tropical regions, and more specifically in urban and semi-urban areas.

Objective To study the relationship of dengue fever and climate factors. Methods The confirmed dengue infections in hospital cases were detected through the hospital information system. Monthly data for total rainfall, temperature and relative humidity for the year 2010 were obtained from Meteorological Department of Karachi.

Results Overall, 576 cases were positive for dengue and hospitalised during 2010. The mean age was $30 \pm 17.66$ years and $391(67.9 \%)$ were males. Out of 576 cases, 476 (82.6\%) were adults. Dengue infection cases were reported during warmest weather with maximum number of cases $226(39.2 \%)$ reported in the month of October 2010 followed by 135 (23.4\%) in the month of September 2010. The difference between number of positive cases during different months was significant $(p=0.03)$.

Conclusion These results suggest that climate factors such as temperature and rainfall may be responsible for an outbreak of dengue infection. Dengue viruses have a known transmission cycle, but changes in temperature or rainfall may have varied local effects.

\section{P2-364 CLIMATE CHANGE THREATENS BLOOD SUPPLY THROUGH ALTERING THE DISTRIBUTION OF VECTOR-BORNE DISEASE: AN AUSTRALIAN CASE-STUDY}

doi:10.1136/jech.2011.142976k.95

\footnotetext{
1,2 H Bambrick, * ${ }^{2} \mathrm{R}$ Woodruff, ${ }^{2} \mathrm{H}$ Hanigan. ${ }^{1}$ School of Medicine, University of Western Sydney, New South Wales, Australia; ${ }^{2}$ National Centre for Epidemiology and Population Health, Canberra, Australia
}

Introduction Climate change is expected to promote more intense and prolonged outbreaks of vector-borne disease, and alter the geographic boundaries of transmission. This has implications for the safety and supply of fresh blood products around the world. In Australia, a recent outbreak of dengue fever caused a prolonged regional shortage in the supply of fresh blood products. Climate change thus has the potential to affect the safety and supply of blood globally through its impact on vector-borne disease. We demonstrate this using the example of dengue in Australia.

Methods Using four climate change scenarios we modelled geographic regions in Australia suitable for dengue transmission and the number of people living in transmission zones, and estimated the effect of future outbreaks on blood supply.

Results Geographic regions with climates that are favourable to dengue transmission could expand to include large population centres in a number of currently dengue-free regions in Australia and reduce blood supply across several states.

Conclusion Without significant global greenhouse gas reduction, there could be an eightfold increase in the number of people living in dengue prone regions in Australia by the end of the century. Similar impacts will be experienced elsewhere and for other vector-borne diseases, with regions currently on the margins of transmission zones most affected. Globally, climate change is likely to compound existing problems of blood safety and supply in already endemic areas and cause future shortages in fresh blood products through its impact on transmission of vector-borne disease.

\section{P2-365 CLIMATE CHANGE IMPACTS ON ROSS RIVER VIRUS IN AUSTRALIA}

doi:10.1136/jech.2011.142976k.96

${ }^{1,2} \mathrm{H}$ Bambrick, ${ }^{2} \mathrm{R}$ Woodruff. ${ }^{1}$ School of Medicine, University of Western Sydney, New South Wales, Australia; ${ }^{2}$ National Centre for Epidemiology and Population Health, The Australian National University, Canberra, Australia

Introduction Ross River virus disease is the most prevalent mosquito-borne disease in Australia, with 4400 cases annually. We explore how changing future climate, in combination with social responses, may alter the habitat and survival of vectors and hosts. Subsequent changes to the pattern and distribution of infections suggest a range of adaptation strategies for reducing transmission.

Methods We map projected changes to rainfall across Australia to 2100 under "dry" and "wet" scenarios and hypothesise how these changes may affect disease distribution over space and time.

Results Changes to transmission patterns will be regionally-specific. Increasing average temperatures will support virus activity in new regions, or for longer periods of each year, as long as humidity remains sufficient for vector survival. Outbreak patterns will change in some regions as increasing drought is punctuated by heavier rainfalls. Explosive outbreaks between periods of inactivity are increasingly likely in some areas. Epidemic regions bordering endemic regions may move towards endemicity. In coastal areas, saltwater vector breeding will be enhanced by increased tidal inundation with sea level rise. With population growth the number of people at risk of infection will increase each year.

Conclusion The pattern of infections in some local areas may change significantly from the historical norm as climate changes, while local adaptations, such as those to manage water deficit, may inadvertently increase vector habitat. Responses that are coordinated between state and local governments and across portfolios will be most successful and cost effective. 